\title{
Freeze-dried versus fresh platelet-rich plasma in acute wound healing of an animal model
}

\author{
Stephany C Huber*,1, José Luiz R C Junior ${ }^{1}$, Letícia Q Silva ${ }^{1}$, Silmara A L Montalvão ${ }^{1}$ \& Joyce \\ M Annichino-Bizzacchi ${ }^{1}$ \\ ${ }^{1}$ Hemocentro, Haemostasis Laboratory, State University of Campinas - UNICAMP, 13083-970, Campinas, São Paulo, Brazil \\ *Author for correspondence: stephany_huber@yahoo.com.br
}

\begin{abstract}
Aim: To compare freeze-dried and fresh platelet-rich plasma (PRP) preparations, in a pre-clinical study. Materials \& methods: 30 Wistar male rats were used to compare and characterize human PRP which was applied at the perilesional area in an acute wound model, evaluated by macroscopical and histological analysis. Results: Despite the increased growth factor concentration after the freeze-drying process, no change in the healing kinetics was observed in vivo. Nevertheless, a significant increased number of myofibroblasts was demonstrated in comparison with the fresh PRP group. We also demonstrated a significant increased percentage of blood vessels in comparison with controls in both the superficial and deep epidermis. Conclusion: These results encourage randomized clinical trials to evaluate the effectiveness of freeze-dried PRP for skin ulcer treatment.
\end{abstract}

First draft submitted: 13 September 2018; Accepted for publication: 17 April 2019; Published online:

22 May 2019

Keywords: blood vessels $\bullet$ freeze-dried platelet-rich plasma $\bullet$ myofibroblasts $\bullet$ platelet-rich plasma $\bullet$ wound healing

With the increase in prevalence of obesity and diabetes, there is currently a simultaneous increase in chronic wounds and tissue repair defects. Chronic wounds have a negative impact upon the quality of life of patients and result in high costs for the health system [1,2]. In the EU $2 \%$ of the annual health budget is used for treatment of chronic wounds. 4 months of outpatient treatment costs between 200 and 2000 pounds, and annually 40 million are used to treat ulcers $[3,4]$.

Healing is a complex process which includes hemostasis, inflammation and remodeling, with the aim of restoring a functional barrier. This entire process is regulated by several cell types and amounts of cytokines and/or growth factors. After injury, the growth factors present in macrophages and platelets are released and resulted in an inflammatory phase that influences all aspects of the wound healing process directly or indirectly [5-7]. More than 30 cytokines which play a central role in wound healing, have been detected in platelets, such as: VEGF, EGF, PDGF, TGF- $\beta$ and IGF $[8,9]$. Due to the potential of improving wound healing, through molecular and cell induction responses, the use of platelet concentrates has been gaining attention $[10,11]$. Platelet-rich plasma (PRP) is defined as platelets concentrated over the basal number (four- to nine-fold) in a small plasma volume [12]. Until now, a number of different techniques to prepare PRP, including commercial systems and in house preparations, have been described. There is no consensus regarding time and speed of centrifugation, use or not of activators and leukocytes in the PRP preparations which lead to a heterogeneity of results in PRP studies. An attempt in order to standardize heterologous PRP products was made by Muraglia et al. focused on culture medium supplement, improving the proliferation, with genetic stability of cells [13,14]. Furthermore, Spanó et al. used similar methodology for in vitro and in vivo tests, in biological membranes with positive results for wound healing [11].

Some studies using PRP to treat ulcers showed no effectiveness $[15,16]$ however others have demonstrated improvement in vascularization, decrease in wound area and rapid epithelization, contributing to an improvement in healing $[17,18]$. These studies used different protocols, based on in house techniques, and only determined platelet counts. However, the concentration of growth factors is also of great importance in the PRP context and a protocol capable of promoting a high number of platelets and growth factor concentration becomes valuable. Thus, in terms of medium culture supplement, Muraglia et al. published a standardized protocol showing functional action for culture of mesenchymal stem cells derived from bone marrow, from adipocyte and umbilical cord. They

Future Medicine 
evaluated the number of platelets, concentration of growth factors, including freeze-dried PRP (FD-PRP) and time of storage [13,14]. Our group recently published a study showing the maintenance of concentration and functional parameters of growth factors of PRP after freeze-dried process in cell culture experiments [19].

The use of FD-PRP can be interesting in clinical practice due to the facility of being ready to use after hydration. Pietramaggiori and colleagues compared treatment of chronic wounds in rats with human fresh frozen or FD-PRP and controls. PRP preparations increased granulation tissue in comparison to controls, spurring new ideas in the regenerative medicine field [20]. The possible use of growth factors derived from platelets, and the potential to prolong the shelf-life of platelet concentrates by freeze-drying becomes a particularly suitable method for improving wound healing treatment, as demonstrated the maintenance of function by in vitro tests $[13,14,19]$.

This study aims to advance in the knowledge of healing, offering a biological mean of accelerating the healing process using PRP. As a preclinical study we compared two forms of PRP preparations, fresh and FD-PRP.

With this study we hope to assist to improve the therapeutic approach, offering an aid to treatment based on evidence to be used in clinical practice, in the healing of nonhealing ulcers, contributing to greater quality of life of patients and a decrease in public health costs.

\section{Methodology}

\section{Fresh PRP}

Two platelet concentrate bags $(60 \mathrm{ml}$ each) from a single donor were obtained from the blood bank (Hemocentro of the University of Campinas - Unicamp). The platelet concentrate was transferred to falcon sterile tubes and centrifuged at $700 \mathrm{x}$ g for $17 \mathrm{~min}$, as previously described [21]. $80 \%$ of the plasma volume was discarded and $20 \%$ was resuspended as PRP. Aliquots were used to quantify platelet number in a hematological counter (CellDyn) and for growth factors quantification. $1 \mathrm{ml}$ was used for treatment as fresh PRP and the remaining volume was used for FD-PRP.

\section{FD-PRP}

In sterile vials for lyophilization, $440 \mu \mathrm{l}$ of fresh PRP was mixed with $20 \mu \mathrm{l}$ of each solution in a final concentration of Tris $0.05 \mathrm{M}$, glycine $0.06 \mathrm{M}$ and sucrose solution $0.06 \mathrm{M}$, totaling $60 \mu \mathrm{l}$ and homogenized. The vials were placed on the shelf of the lyophilizer, which was stored at $-80^{\circ} \mathrm{C}$ for $2 \mathrm{~h}$ to freeze the samples. After $2 \mathrm{~h}$, the shelf was placed in the lyophilizer, reaching the temperature and vacuum programmed, and the lyophilization process occurred for $19 \mathrm{~h}$. After the process the vials were stored at $-20^{\circ} \mathrm{C}$. This protocol was based on a previous standardization lyophilization study of our group published by da Silva et al. [19]. For the experiments the FDPRP was reconstituted with sterile water at the same volume used before the lyophilization $(500 \mu \mathrm{l})$ and injected according to the experimental method.

\section{Quantification of growth factors in PRP}

Growth factors were quantified in FD-PRP and fresh PRP by multiplex technology (Luminex - Millipore). The growth factors analyzed were: EGF, PDGF-AA, TGF- $\beta 1$ and VEGF. The protocol was conducted according to the manufacture's procedure.

\section{Experimental animals}

30 Wistar male rats were obtained from the Multidisciplinary Center for Biological Research in the Area of Science in Laboratory Animals (CEMIB), Unicamp, SP, Brazil. During the experiments, there were two animals per cage, separated by a steel partition, avoiding, thus, the contact between the rats. The animals were maintained under standard conditions of lighting (cycle of $12 \mathrm{~h} \mathrm{light/dark)} \mathrm{and} \mathrm{temperature} \mathrm{of} 22 \pm 2{ }^{\circ} \mathrm{C}$. The experiments were performed with rats with 6-8 weeks of age, and all the animals received diet and water ad libitum. The procedures were performed in conformity with the Brazilian laws of animal experimentation approved by the Ethical Commission for Animal Use (CEUA-Unicamp).

After intramuscular anesthesia with ketamine $(75 \mathrm{mg} / \mathrm{kg}$; Ketalar, Parke-Davis, Brazil) and xilazine $(10 \mathrm{mg} / \mathrm{kg}$; União Química Farmacêutica Nacional S/A, Brazil) the animals were placed in prone position and, after shaving the dorsal region, a wound of $1 \mathrm{~cm}^{2}$ was made in the midline with the aid of a scissor. The animals were divided into three groups of ten according to treatment: Control - saline, fresh PRP - PRP, and FD-PRP - FD-PRP. We use different animals for each treatment as described by other authors [22]. The use of the same animal with multiple 
Table 1. Levels of growth factors in fresh and freeze-dried platelet-rich plasma.

\begin{tabular}{|llll}
\hline Growth factor $(\mathbf{p g} / \mathbf{m l})$ & Freeze-dried PRP & Fresh PRP & Fold dried/fresh \\
\hline PDGF-AA & 192571.0 & 51783.2 & 3.72 \\
\hline VEGF & 2428.2 & 1477.9 & 1.64 \\
\hline EGF & 3595.5 & 1316.9 & 2.73 \\
\hline TGF & 4138.8 & 1609.1 & 2.57 \\
\hline PRP: Platelet-rich plasma. & & &
\end{tabular}

lesions is questionable as each lesion could be exposed to different stimuli, according to the site in the body. In order to minimize this variability, the same animal was used and the number of animals in each group was increased.

The method of application consisted of perilesional injections of $500 \mu$ l of PRP (fresh or FD) or saline using a syringe and needle of $24 \mathrm{G}$ in the day of the wound induction (D0). No cover was used.

\section{Wound closure monitoring}

To evaluate wound contraction, a transparent plastic film was placed over the lesion and wound margins were traced at specific time-points: days 0, 3, 7 and 10, as previously standardized [22,23]. After digitalization, the wound area was measured using Image J software (MD, USA). Wound area measurements and wound photographs were obtained on these specific days, until euthanasia, without scab removal. Wound sizes were expressed as percentage of the initial wound area; in this way, the value in $\mathrm{cm}^{2}$ in the day 0 was considered $100 \%$ and the other days were measured in relation to day 0 , as follows:

$$
\begin{aligned}
& \text { Day } 0\left(\mathrm{~cm}^{2}\right)-100 \% \\
& \text { Day } 3\left(\mathrm{~cm}^{2}\right)-\mathrm{X}
\end{aligned}
$$

\section{Histological \& immunohistochemistry evaluation of wound healing}

Euthanasia was performed ten days after wounding using deep anesthesia. The ulcerated area and adjacent normal skin were excised, formalin-fixed and paraffin-embedded. $5 \mu \mathrm{m}$ sections were stained with hematoxylin-eosin and Masson's trichrome for the evaluation of the characteristics of the epithelia and collagen density.

Epithelial thickness was evaluated in Masson's trichrome, using ten pictures obtained with a microscope $\mathrm{x} 10$ objective. Each picture was measured in ten points of the epithelia, totaling 100 measures for slide using Image J software [24].

Collagen density was calculated in Masson's trichrome with the evaluation of three pictures for each slide (x10 objective). Each picture was measured using the software Image J, option color deconvolution in an area of $10,000 \mu \mathrm{m}^{2}$ of dermis. Results were expressed as density of collagen $/ \mu \mathrm{m}^{2}[25]$.

Immunohistochemistry was performed by heat antigenic recovery, with a monoclonal antibody against $\alpha$-smooth muscle actin ( $\alpha$-SMA, clone 1A4, 1:300, DBS, CA, USA), to identify functional blood vessels (arterioles) and myofibroblasts. Three animals per group were randomly chosen. The quantification of blood vessels and myofibroblasts were performed through the evaluation of five random fields per animal on the superficial region, and five random fields in the deep region of the granulation tissue using a $\mathrm{x} 20$ objective lens in a video microscopic system (Nikon microscope, Infinity 1 video camera) and expressed as the percentage of area. Blood vessels were determined as number of vessels/field.

\section{Statistics}

Comparative analyses were performed by Kruskal-Wallis test with Dunns post-test to compare all pairs of columns. The significance level adopted for the statistical tests was 5\% ( $\mathrm{p} \leq 0.05)$. Analyses were performed with using GraphPad, version 5 .

\section{Results}

\section{PRP characterization}

Fresh and FD-PRP were characterized according to platelet number and growth factor concentration. The platelet number of fresh PRP was $5714 \times 10^{3}$ cells/ $\mu$ l, and the concentrations of growth factors are shown in Table 1 . 


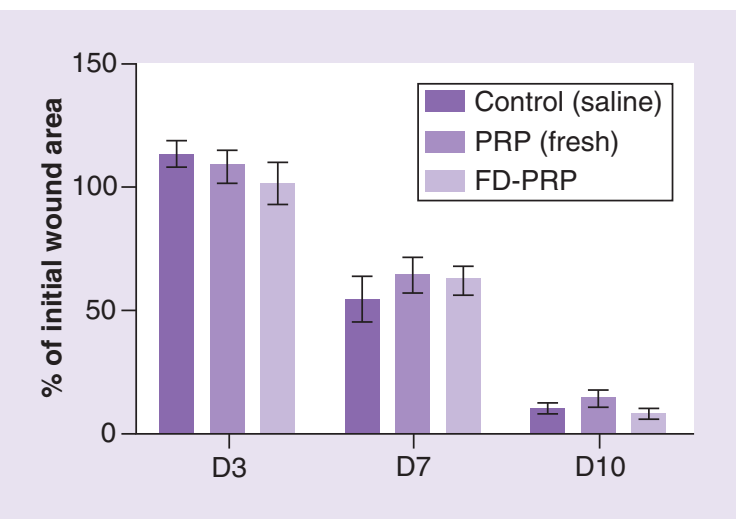

Figure 1. Kinetics of wound closure in percentage of initial area of the different treatments during days 3 (D3), 7 (D7) and 10 (D10) of experiments. FD-PRP: Freeze-dried PRP; PRP: Platelet-rich plasma.
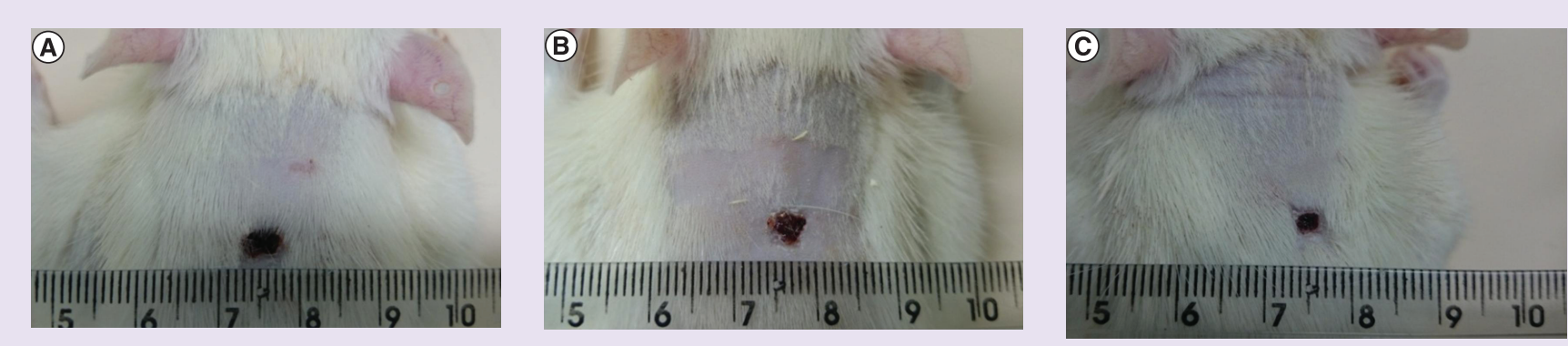

Figure 2. Macroscopic photographic of the wounds on day 10.

(A) Control (saline), (B) PRP, (C) FD-PRP.

FD-PRP: Freeze-dried PRP; PRP: Platelet-rich plasma.

The FD-PRP presented higher concentration of all growth factors when compared with fresh PRP from 1.64 to 3.72-fold. Platelet number after freeze-drying process was $268 \times 10^{3}$ cells/ $\mu$, which represents a loss of $95.3 \%$ of platelet number.

\section{Wound closure analysis}

Figure 1 represents the mean and standard deviation of initial wound area expressed as percentage, measured 3 (D3), 7 (D7) and 10 (D10) days after wounding.

The mean and standard deviation $(\mathrm{SD})$ of area on $\mathrm{D} 3$ was $112.9 \pm 16.6 \%, 107.9 \pm 21.3$ and $100.8 \pm 30.2$ for control, PRP and FD-PRP, respectively. On D7 the areas were 54.3 $\pm 28.9,63.9 \pm 22.8$ and $61.6 \pm 20.6$ for control, PRP and FD-PRP, respectively. On D10 the results were $9.9 \pm 6.1,13.9 \pm 11.2,7.7 \pm 6.0$ for control (saline), PRP and FD-PRP, respectively. Figure 2 demonstrates macroscopic photographic of the wounds at D10. We observed, though not statistically significant, a trend to smaller wound areas with FD-PRP.

\section{Histological analysis Epithelial thickness}

Figure 3 represents epithelial thickness in different groups at D10. The mean and SD of epithelial thickness was $25.37 \pm 7.63 \mu \mathrm{m}$ for control, $24.56 \pm 7.21 \mu \mathrm{m}$ for PRP and $25.52 \pm 5.84 \mu \mathrm{m}$ for FD-PRP (not statistically significant, $\mathrm{p}=0.08876$ ).

\section{Collagen density}

The mean and SD of collagen density at day 10 (D10) was $183.6 \pm 32.59$ pixels $/ \mu \mathrm{m}^{2}$ for control, $174.6 \pm 38.65$ pixels $/ \mu \mathrm{m}^{2}$ for PRP and $193.8 \pm 25.36$ pixels $/ \mu \mathrm{m}^{2}$ for FD-PRP, as shown in Figure 4 (not statistically significant, $\mathrm{p}=0.5679$ ). 
Figure 3. Epithelial thickness of the different treated groups measured by Masson's trichrome on day 10. FD-PRP: Freeze-dried PRP; PRP: Platelet-rich plasma.
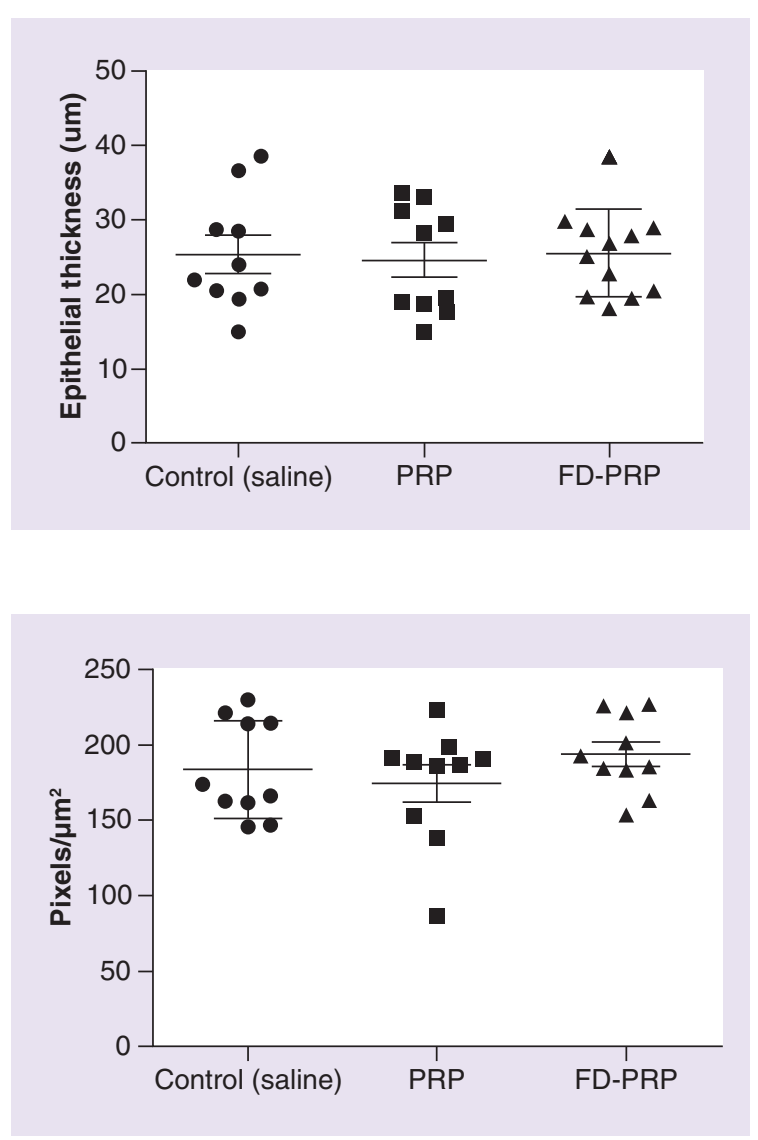

Figure 4. Collagen density by Masson's trichrome of the treated groups on day 10.

FD-PRP: Freeze-dried PRP; PRP: Platelet-rich plasma.
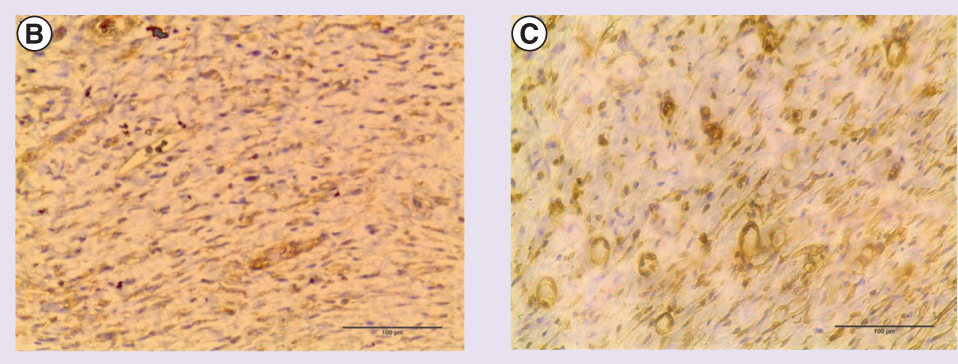

Figure 5. Image of imunohistochemistry marked with anti- $\alpha$ actin for blood vessels and myofibroblasts in the skin. (A) Control (saline), (B) platelet-rich plasma, (C) freeze-dried platelet-rich plasma.

\section{Myofibroblasts through imunohistochemistry}

Figure 5 shows the quality of imunohistochemistry image for all the groups.

Quantification of myofibroblasts by area in imunohistochemistry using Image J software showed that the percentage was higher with FD-PRP treatment when compared with the other groups, in deep and superficial epidermis. This increase of myofibroblasts was statistically different when comparing FD-PRP and PRP treatments $(\mathrm{p}=0.0095)$ in deep region of epidermis at D10 (Figure 6).

\section{Blood vessel density}

Our results obtained by imunohistochemistry showed an increased percentage of blood vessels per area in the group treated with FD-PRP when compared with controls, both in deep and superficial epidermis at D10, as demonstrated in Figure 7. 
(A)

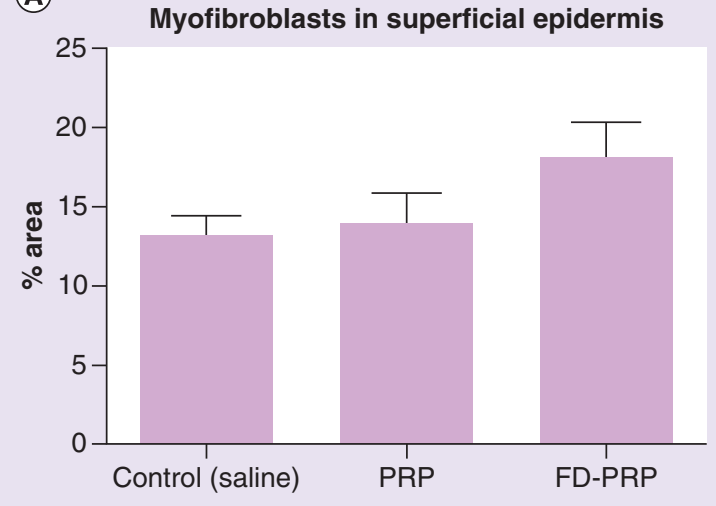

(B)

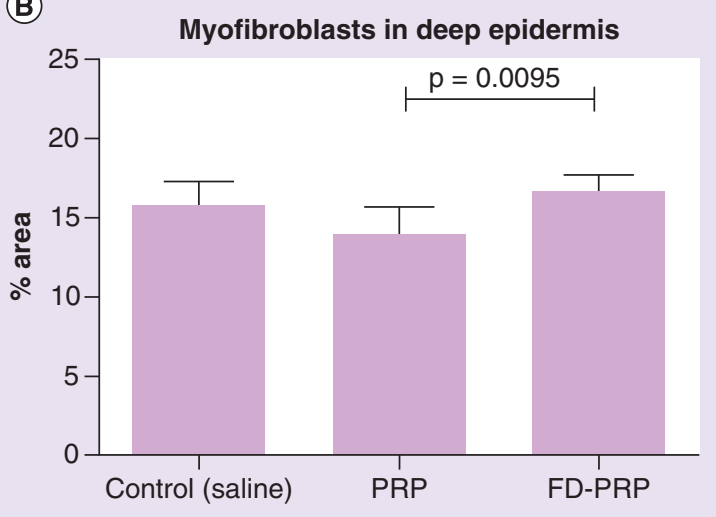

Figure 6. Quantification of myofibroblasts using imunohistochemistry with smooth $\alpha$-actin antibody and treated by Image J software, evidencing the mean and standard deviation of treated groups. (A) Superficial epidermis, (B) deep epidermis.

FD-PRP: Freeze-dried PRP; PRP: Platelet-rich plasma.

(A)

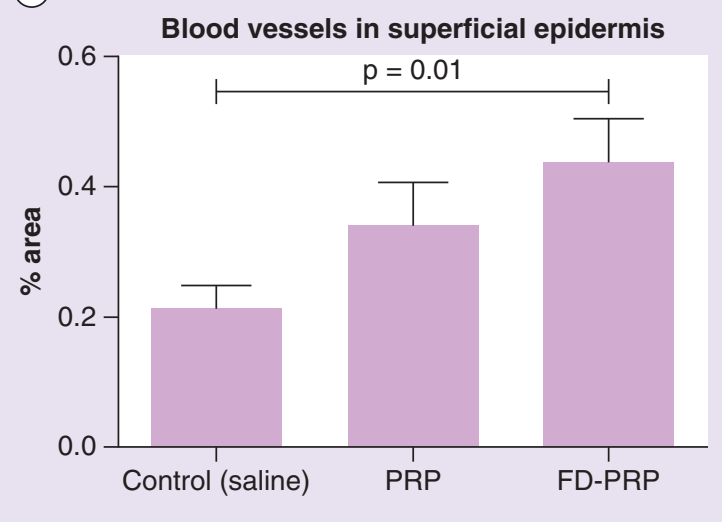

(B)

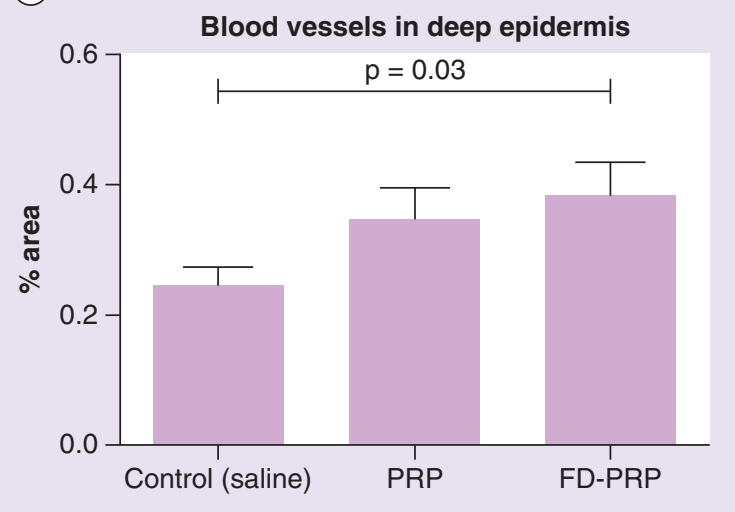

Figure 7. Quantification of blood vessels by imunohistochemistry using smooth $\alpha$-actin antibody and treated by Image J software, evidencing the mean and standard deviation and coefficient of variation of the treated groups. (A) Superficial epidermis, (B) deep epidermis.

FD-PRP: Freeze-dried PRP; PRP: Platelet-rich plasma.

\section{Discussion}

The reason for the use of the term FD-PRP is that this terminology is used in the majority of the previously published articles [10,19]. We understand that the lyophilization process damages approximately $40-90 \%$ of the platelets, however not all of them. Moreover, the action of PRP is mainly derived from the growth factors and not from the platelets. In this way, it was observed an increase in the growth factors concentration, which was an expected result due to the platelet damage caused by lyophilization, with platelets lysis and consequent release of growth factors, increasing the concentration of these proteins.

In our study the application of PRP or FD-PRP did not significantly induce changes in wound healing kinetics measured as contraction when compared with controls during all evaluated days $[3,7,10]$. Though not significantly different, FD-PRP presented the smallest area in almost all days except for D7. Despite epithelial thickness and collagen density not differing among the groups, blood vessels density in percentage of area was significantly higher in those treated with FD-PRP in comparison to the controls, both in superficial and deep regions of epidermis. One relevant result of this study was the quantification of growth factors in PRP, and we believe that this finding is a result of the high concentration of VEGF present in FD-PRP and delivered to the wound area. VEGF plays a 
central role in promoting angiogenesis during wound repair and previous studies demonstrated accelerated wound closure with collagen-binding VEGF topically [26].

The use of PRP is widely used for wound treatment; however, this use remains controversial, with preclinical experiments showing different results and no consensus regarding the use of PRP until now. Mehrjerdi et al. (2008) evaluated the topical effect of autologous PRP without leukocytes versus saline in the healing of dog wounds [15]. Macroscopic and histological evaluations demonstrated no differences in the lesion area between both treatments. Indirect collagen (hydroxyproline) and histological evaluation, demonstrated a trend, though not statistically significant, of increase of the re-epithelization with PRP treatment. These results suggested that PRP did not accelerate or improve the quality of tissue repair with topical use in the treatment of animal acute wounds [11]. Ostvar et al. evaluated the use of PRP without leukocytes in chronic wounds of rabbits compared with saline, with a cover of polyurethane film daily. Through macroscopic and histological evaluation they showed that the healing time of the group treated with PRP was shorter, associated to an increase in the mean of vascular density, demonstrating positive effects in wound healing [18]. Spanò et al. evaluated two bio membranes of PRP, with different concentrations of platelets, for chronic wound regeneration. In an in vitro model the proliferation of keratinocytes, and mesenchymal stem cells derived from bone marrow was evaluated by measuring the level and kinetics of growth factors. Although both bio membranes induced wound healing, a higher platelet concentration resulted in a slowdown of the membrane resorption that interfered with the skin healing [11].

The use of lyophilized or frozen platelets has been studied due to the limitation of short half-life of platelet concentrate. In this way, the search for methods to improve platelet stabilization using biochemicals to preserve their biological activities has acquired great value $[13,14,27,28]$, however the interference of lyophilization in platelet hemostatic function remains questionable [29].

PRP is currently being investigated for a wide range of therapeutic indications, focusing on the growth factors and cytokines present in PRP granules. For this means FD-PRP could be an interesting approach, based on the ability to maintain a stable preparation, with a single blood collection for multiple samples of PRP that can allow multiple applications. Indeed, we produced an autologous product with high concentration of growth factors. This is of great value in clinical practice, however there is little basic and preclinical research regarding this area.

Pietramaggiori and colleagues contributed to the field of freeze-dried and wound healing. The better effectiveness of lyophilized PRP with and without stabilizing agents in the treatment of wounds of diabetic mice compared with control was evidenced by an increased area of granulation tissue in the former. There was no difference in the concentration of growth factors (PDGF-AB, TGF- $\beta 1$, VEGF and EGF) among the different preparations, nevertheless the in vitro analysis showed an increase in cellular proliferation with lyophilized PRP [13,14,19]. Another experiment comparing single or multiple injection applications in the wound area of diabetic mice with FD-PRP containing trehalose, fresh PRP, multiple doses of recombinant VEGF or no treatment demonstrated multiple doses of VEGF and the use of FD-PRP promoted a faster wound closure when compared with the nontreated group [30]. Promising results with higher proliferation rate, revascularization and better re-epithelization for diabetic wound healing have been described with the use of micronized dermis in association with FD PRP [31].

Regarding the induction of differentiation into myofibroblasts with the use of PRP, the results in literature remain controversial. TGF- $\beta$ is a well-known key molecule inducer for myofibroblastic differentiation. However, PRP is a complex mixture including other molecules that can counteract this myofibroblast inductive-effect. The induction was verified by authors that use PRP rich in leukocytes (L-PRP) $[32,33]$. Anitua et al. $(2013,2012)$ did not show this differentiation with P-PRP (called PRGF) in vitro, concluding that the differences found regarding myofibroblasts could be due to the different PRP composition in terms of leukocyte content $[34,35]$. In our study, we showed an increase in the concentration of myofibroblasts with the use of FD-PRP, and we believe that this effect was achieved due to the high concentration of growth factors induced by lyophilization process.

Some studies have suggested a positive influence of the concentrate platelets on angiogenesis. Notodihardjo et al. used the PRP releasate (PRPr) impregnated in a biodegradable hydrogel gelatin for wound healing in mice. After 14 days of wounding, an increase in the number of capillary formation was verified [9]. Cheng et al. also reported an increase in the number of new blood vessels in the group treated with PRP for hair growth [36]. The increase in blood vessels showed in our study corroborate previous articles, suggesting that the significant increase of blood vessels in the group treated with FD-PRP is due to the high concentration of growth factors induced by lyophilization.

One limitation of this study was the acute ulcer model, and we could not rule out the possibility of a better impact in treatment of chronic wounds. 


\section{Conclusion}

In conclusion, results obtained with the treatment of acute ulcer in rats with PRP or FD-PRP point towards lyophilization as a good option in clinical practice. This is very interesting especially for those patients who require multiple applications.

Increased VEGF concentration in FD-PRP may be related to higher vascularization demonstrated with this treatment, which is of great value for wound healing.

\section{Translational perspective}

Since the use of PRP for the treatment of wounds has been showing promise results, the possibility of increasing the shelf-life of PRP products with lyophilization is of great interest, especially for patients who require multiple applications. The use FD-PRP is practical, safe and of great interest for clinical practice. The next step is the evaluation in clinical trials for wound healing of different etiologies.

\section{Summary points}

- The use of platelet-rich plasma (PRP) is suitable for wounds due to the presence of multiple cytokines with important roles in regeneration/ healing.

- The freeze-drying process is a possibility to use in clinical practice to enhance the shelf-life of PRP products.

- Characterization of PRP products is realized through platelet number and growth factors concentration.

- In vivo evaluation of PRP function is conducted in fresh, and after the freeze-drying process.

- Macroscopic evaluation of wound healing is done through kinetics of wound closure according to time.

- Histologic evaluation of PRP products is performed by the epithelial thickness, collagen density and immunohistochemistry for myofibroblasts and blood vessels in deep and superficial epidermis - to evaluate the healing process of the different PRP products.

- A significant increase in blood vessels and myofibroblasts number when the animals were treated with freeze-dried PRP demonstrated promising results.

- Encouraging for clinical trials due to the increase of vascularization, the freeze-drying process represents an option for patients who require multiple applications; maintaining the properties of fresh PRP and showing positive results.

Author contributions

SC Huber was the principal investigator, responsible of the project and animal manipulation. SAL Montalvão was part of the study design, performed statistical analysis and reviewed and correct the manuscript. JL Cunha, SC Huber and LQ Silva were presented in the technical performance, such as the growth factors quantification and buffers standardization. JM Annichino-Bizzacchi guided this project, drafting, revising and finalizing the manuscript.

\section{Acknowledgments}

The authors would like to thank the Experimental Pathology Laboratory for the support of histological analysis and procedure. We are thankful to R Foglio for correcting our English in the manuscript.

\section{Financial \& competing interests disclosure}

This study was supported by grants from the National Institute of Science and Technology of Blood, Brazil (INCT do Sangue CNPq/MCT/FAPESP) and FAPESP. The authors have no other relevant affiliations or financial involvement with any organization or entity with a financial interest in or financial conflict with the subject matter or materials discussed in the manuscript apart from those disclosed.

No writing assistance was utilized in the production of this manuscript.

Ethical conduct of research

The procedures were performed in conformity with the Brazilian laws of animal experimentation. Ethical Commission for Animal Use (CEUA-Unicamp) approved of the experimental procedures (CPEA:2290-1). 
Availability of data \& material

The datasets used and/or analyzed during the current study are available from the corresponding author on reasonable request.

\section{References}

Papers of special note have been highlighted as: $\bullet$ of interest; $\bullet \bullet$ of considerable interest

1 Scherer SS, Tobalem M, Vigato E et al. Nonactivated versus thrombin-activated platelets on wound healing and fibroblast-to-myofibroblast differentiation in vivo and in vitro. Plast. Reconstr. Surg. 129(1), 46e-54e (2012).

2 Ferreira AM, Mattu C, Ranzato E, Ciardelli G. Bio-inspired porous membranes containing polymer nanoparticles for wound healing. J. Biomed. Mater. Res. A 102(12), 4394-405 (2014).

3 Crovetti G, Martinelli G, Issi M et al. Platelet gel for healing cutaneous chronic wounds. Transfus. Apher. Sci. 30(2), 145-151 (2004).

4 Anitua E, Aguirre JJ, Algorta J et al. Effectiveness of autologous preparation rich in growth factors for the treatment of chronic cutaneous ulcers. J. Biomed. Mater. Res. B. Appl. Biomater. 84(2), 415-21 (2008).

5 Gonzalez ACO, Andrade ZA, Costa TF, Medrado ARPM. Wound healing - a literature review. An. Bras. Dermatol. 91(5), 614-20 (2016).

6 Golebiewska EM, Poole AW. Platelet secretion: From haemostasis to wound healing and beyond. Blood Reviews. 29(1), 153-162 (2015).

7 Han G, Ceilley R. Chronic wound healing: A review of current management and treatments. Adv. Ther. 34(1), 599-610 (2017).

8 Ahmed M, Reffat SA, Hassan A, Eskander F. Platelet-rich plasma for the treatment of clean diabetic foot ulcers. Ann. Vasc. Surg. 38(1), 206-2011 (2016).

9 Notodihardjo PV, Morimoto N, Kakudo N et al. Comparison of the efficacy of cryopreserved human platelet lysate and refrigerated lyophilized human platelet lysate for wound healing. Regen. Med. 10(1), 1-9 (2019).

10 Morimoto N, Kakudo N, Ogura T et al. Easy-to-use preservation and application of platelet-rich plasma in combination wound therapy with a gelatin sheet and freeze-dried platelet-rich plasma: a case report. Eplasty. 16(1), 202-207 (2016).

11 Spanò R, Muraglia A, Todeschi MR et al. Platelet-rich plasma-based bioactive membrane as a new advanced wound care tool. J. Tissue Eng. Regen. Med. 12(1), e86-e96 (2018).

12 Marx RE. Platelet-rich plasma: evidence to support its use. J. Oral Maxillofac. Surg. 62(4), 489-496 (2004).

13 Muraglia A, Ottonello C, Spanò R et al. Biological activity of a standardized freeze-dried platelet derivate to be used as cell culture medium supplement. Platelets 25(3), 211-220 (2014).

14 Muraglia A, Nguyen VT, Nardini M et al. Culture medium supplements derived from human platelet and plasma: cell commitment and proliferation support. Front. Bioeng. Biotechnol. 5(1), 1-15 (2017).

15 Mehrjerdi HK, Sardari K, Emami MR et al. Efficacy of autologous platelet-rich plasma (PRP) activated by thromboplastin-D on the repair and regeneration of wounds in dogs. Iran J. Vet. Surg. 3(4), 19-30 (2008).

16 Neto NMA, Del Carlo RJ, Monteiro BS et al. Role of autologous mesenchymal stem cells associated with platelet-rich plasma on healing of cutaneous wounds in diabetic mice. Clin. Exp. Dermatol. 37(5), 544-553 (2012).

17 DeRossi R, Coelho ACAO, Mello GS et al. Effects of platelet-rich plasma gel on skin healing in surgical wound in horses. Acta Cir. Bras. 24(4), 276-281 (2009).

18 Ostvar O, Shadvar S, Yahaghi E et al. Effect of platelet-rich plasma on the healing of cutaneous defects exposed to acute to chronic wounds: a clinic-histopathologic study in rabbits. Diagn. Pathol. 10(1), 85-91 (2015).

19 da Silva LQ, Montalvão SAL, Justo-Junior AS et al. Platelet-rich plasma lyophilization enables growth fator preservation and functionality when compared with fresh platelet-rich plasma. Regen. Med. 13(7), 775-784 (2018).

20 Pietramaggiori G, Kaipainen A, Czeczuga JM, Wagner CT, Orgill DP. Freeze-dried platelet-rich plasma shows beneficial healing properties in chronic wounds. Wound Repair Regen. 14(5), 573-580 (2006).

21 Amable PR, Carias RBV, Teixeira MVT et al. Platelet-rich plasma preparation for regenerative medicine: optimization and quantification of cytokines and growth factors. Stem Cell Res. Ther. 4(3), 67-80 (2013).

-. Standardization of platelet-rich plasma (PRP) products based in this article, complete characterization of PRP.

22 Romana-Souza B, Nascimento AP, Monte-Alto-Costa A. Low-dose propranolol improves cutaneous wound healing of burn-injured rats. Plast. Reconstr. Surg. 122(6), 1690-1699 (2008).

-. Standardization of wound healing evaluation.

23 Nascimento AP, Costa AM. Overweight induced by high-fat diet delays rat cutaneous wound healing. Br. J. Nutr. 96(6), 1069-1077 (2006).

- Histologic and macroscopic technique for in vivo assays.

24 Jensen EC. Quantitative analysis of histological staining and fluorescence using ImageJ. Anat. Rec. 296(3), 378-381 (2013).

25 Ruifrok AC, Johnston DA. Quantification of histochemical staining by color deconvolution. Anal. Quant. Cytol. Histol. 23(4), 291-299 (2001). 
26 Yan X, Chen B, Lin Y et al. Acceleration of diabetic wound healing by collagen binding vascular endothelial growth factor in diabetic rat model. Diabetes Res. Clin. Pract. 90(1), 66-72 (2010).

27 Vostal JG, Mondoro TH. Liquid cold storage of platelets: a revitalized possible a alternative for limiting bacterial contamination of platelet production. Transfus. Med. Rev. 11(4), 286-95 (1997).

28 Hoffmeister KM, Josefsson EC, Isaac NA, Clausen H, Hartwig JH, Stossel TP. Glycosylation restores survival of chilled blood platelets. Science 301(12), 1531-4 (2003).

29 Read et al. Proc. Natl. Acad Sci. USA, 92, 397-401, 1995. US. Pat. No. 5,902,608, issued May 11, 1999, US. Pat. No. 5,827,741, Beattie et al., issued Oct. 27, 1998.

30 Pietramaggiori G, Kaipainen A, Ho D et al. Trehalose lyophilized platelets for wound healing. Wound Repair Regen. 15(2), 213-220 (2007).

- $\quad$ Key article for freeze-dried PRP process and evaluation.

31 Pietramaggiori G, Scherer SS, Mathews JC et al. Healing modulation induced by freeze-dried platelet-ruch plasma and micronized allogenic dermis in a diabetic wound model. Wound Repair Regen. 16(2), 218-225 (2008).

32 Kushida S, Kakudo N, Suzuki K, Kusumoto K. Effects of platelet-rich plasma on proliferation and myofibroblastic differentiation in human dermal fibroblasts. Ann. Plast. Surg. 71(2), 219-224 (2013).

33 Giovanini AF, Gonzaga CC, Zielak JC et al. Platelet-rich plasma (PRP) impairs craniofacial bone repair associated with its elevated TGF- $\beta$ levels and modulates the co-expression between collagen III and $\alpha$-smooth muscle actin. J. Orthop. Res. 29(3), 457-463 (2011).

34 Anitua E, Muruzabal F, Alcalde I, Merayo-Lloves J. Plasma rich in growth factors (PRGF-Endoret) stimulates corneal wound healing and reduces haze formation after PRK surgery. Exp. Eye Res. 115(1), 153-161 (2013).

35 Anitua E, Troya M, Orive G. Plasma rich in growth factors promote gingival tissue regeneration by stimulating fibroblast proliferation and migration and by blocking transforming growth factor-beta1Yinduced myodifferentiation. J. Periodontol. 83(8), 1028-37 (2012).

36 Cheng H, Zhang J, Li J, Jia M, Wang Y, Shen H. Platelet-rich plasma stimulates angiogenesis in mice which may promote hair growth. Eur. J. Med. Res. 22(1), 39-45 (2017). 